\title{
Research and clinical applications of molecular biomarkers in gastrointestinal carcinoma (Review)
}

\author{
FENG JIAO, ZILIANG JIN, LEI WANG and LIWEI WANG
}

Department of Oncology, The First People's Hospital Affiliated to Shanghai Jiao Tong University, Shanghai 201620, P.R. China

Received May 05, 2013; Accepted August 12, 2013

DOI: $10.3892 /$ br.2013.158

\begin{abstract}
Gastrointestinal (GI) carcinoma is a common malignant disease worldwide. Its development and progression is a multistage process involving a multifactorial etiology. Although the detailed mechanisms of the development of GI carcinoma remain controversial, the elucidation of its molecular biology over the last few years has resulted in a better perspective on its epidemiology, carcinogenesis and pathogenesis. More significantly, it is currently possible to use biological indicators or biomarkers in differential diagnosis, prognostic evaluation and specific clinical interventions. In this review, we aimed to describe the biomarkers of pathogenesis, invasion, metastasis and prognosis of GI carcinoma and discuss their potential clinical applications. The majority of these biomarkers, such as tumor-associated antigens, oncogenes and tumor suppressor genes, metastasis-associated genes, cell adhesion molecules, cytokines, growth factors and microRNAs, are currently broadly applicable.
\end{abstract}

\section{Contents}

1. Introduction

2. Tumor-associated antigens (TAAs)

3. Oncogenes and tumor suppressor genes

4. Metastasis-associated genes

5. Cell cycle and apoptosis regulators

6. Cell adhesion molecules

7. Growth factors, cytokines and angiogenic factors

8. Matrix metalloproteinases (MMPs)

9. MicroRNAs

10. Conclusion

Correspondence to: Professor Liwei Wang, Department of Oncology, The First People's Hospital Affiliated to Shanghai Jiao Tong University, 650 New Songjiang Road, Shanghai 201620, P.R. China

E-mail:yzwlw@yahoo.com

Key words: gastrointestinal carcinoma, biomarker, biological indicator

\section{Introduction}

The term 'gastrointestinal (GI) carcinoma' comprises esophageal, gastric, liver, pancreatic and colorectal cancer. In this review, only gastric and colorectal cancer are addressed, both of which are a common type of fatal malignancy worldwide. According to GLOBOCAN 2008 (1), a total of 989,600 new gastric cancer cases and 738,000 cancer-related deaths were estimated to have occurred in 2008, accounting for $8 \%$ of total cancer cases and $10 \%$ of total deaths. Colorectal cancer was the third most commonly diagnosed cancer among males and the second among females, with $>1.2$ million new cancer cases and 608,700 deaths (2). Despite the extensive diagnostic and therapeutic interventions, GI carcinoma remains a major health concern $(3,4)$. It was demonstrated that the development and progression of GI carcinoma is a multistage process, involving several genetic and epigenetic alterations. Alterations in specific genes or proteins that are involved in diverse cell functions, such as cell proliferation, cell apoptosis and metastasis have been identified $(5,6,7)$, but remain to be elucidated. The altered expressions of these genes or proteins during tumorigenesis may be used as biomarkers, which may provide information regarding the molecular events during the initiation and progression of cancer. They may also affect cancer diagnosis and prognosis and ultimately lead to improvements in the treatment and prevention of malignancies. This review focuses on the biological markers of pathogenesis, invasion, metastasis and prognosis in GI carcinoma and their potential clinical applications.

\section{Tumor-associated antigens (TAAs)}

Over the past few years, it was demonstrated that cancer is immunogenic and numerous tumor antigens have been identified in cancer patients. Autoantibodies against TAAs are attractive biomarkers that may be employed as a potential diagnostic and prognostic tool, used for the development of non-invasive serological tests, owing to their specificity and stability in the serum (8). Unlike circulating proteins that are shed by bulky tumors, serum autoantibodies are detectable even when antigen expression is minimal (9). The carcinoembryonic antigen (CEA) was first identified in fetal gut tissue. Subsequently, it was detected in the circulation of colorectal cancer patients and was recognized as a serum marker for colorectal cancer. The determination of preoperative CEA 
serum levels is useful for the diagnosis and prognosis of recurrence and survival in colorectal cancer patients and an increase in the CEA levels is associated with a more advanced tumor stage. Similarly, carbohydrate antigen (CA) 19-9 levels have been shown to increase in the advanced stages of colorectal cancer. Despite its lower sensitivity compared to CEA in the early stages of colorectal cancer, the combination of CEA and CA 19-9 may provide more information compared to CEA alone for the prognosis of recurrence and patient survival (10). In gastric cancer, CEA and/or CA 19-9 monitoring following surgery has been proven useful in the prediction of recurrence, particularly in patients exhibiting high preoperative levels of these markers $(11,12)$. High levels of CA 72-4 in peritoneal washing were significantly correlated with lymph node metastasis, serosal involvement and a more advanced stage of gastric carcinoma (13). The sensitivity of serum CA 72-4 is limited; however, the combination of CEA, CA 19-9 and CA 72-4 considerably improved sensitivity without impairing specificity $(14,15)$. Since a single diagnostic or prognostic biomarker exhibits poor sensitivity, specificity, or other clinical value, the optimal combination of serum markers in order to enhance their clinical values in the management of GI carcinoma remains a major concern.

\section{Oncogenes and tumor suppressor genes}

The mutational activation and/or amplification of several oncogenes has been documented in GI carcinoma. The KRAS gene is one of the most commonly mutated oncogenes associated with colorectal cancer, with codons 12 and 13 of the KRAS gene harboring mutations in $35 \%$ of cases (16). KRAS testing is mandatory if anti-epidermal growth factor receptor (EGFR) therapy is considered for patients with metastatic colorectal cancer. BRAF mutations also appear to be an important negative prognostic factor (17).

An increased MET copy number was shown to be crucial in determining the prognosis of gastric cancer patients (18). Overexpression of the c-erbB2 gene, which encodes a receptor tyrosine kinase, has been associated with prognosis and response to therapy in several solid tumors. It was suggested that c-erbB2 overexpression may be of prognostic significance in patients with metastatic gastric cancer (19).

Ubiquitin C-terminal hydrolase-L1 (UCHL1) belongs to the family of deubiquitinating enzymes, which is involved in the ubiquitin-dependent proteolytic system. The upregulation of UCHL1 has been associated with lymph node metastasis in colorectal cancer. A recent study has suggested that UCHL1 acts as an oncogene and contributes to colorectal cancer progression by activating the $\beta$-catenin/T cell factor (TCF) pathway through its deubiquitinating activity (20).

The p53 gene is one of most important tumor suppressor genes. p53 acts as a potent transcription factor with a fundamental role in the maintenance of genetic stability. A high frequency of p53 mutations, loss of heterozygosity, overexpression of the p53 protein and, consequently, loss of p53 function, are early events in the course of gastric cancer, as well as an important biomarker of the prognosis and treatment response. In addition, single-nucleotide polymorphisms of 553 play a role in the development and prognosis of gastric cancer (21).
The adenomatous polyposis coli (APC) gene, located on chromosome 5q21-22, acts as an antagonist of the Wnt signaling pathway. It was shown to be involved in genetic instability and is frequently deleted in GI carcinomas. A positive correlation was previously reported between a decrease in the APC copy number and tumor progression (22).

Another tumor suppressor gene at 10q23.3, designated as phosphatase and tensin homolog (PTEN), encoding a dual-specificity phosphatase with lipid and protein phosphatase activity, was shown to be crucial in the pathogenesis of a variety of human cancers. Frequent mutations and deletions of PTEN genes have also been detected in GI carcinoma $(23,24)$.

\section{Metastasis-associated genes}

The metastasis-associated 1 (MTA1) gene encodes a protein that was identified in a screen for genes expressed in metastatic cells, particularly in mammary adenocarcinoma cell lines. The expression of this gene has been correlated with metastatic potential. A previous study suggested that the MTA1 protein may have several functions in cellular signaling, chromosome remodeling and transcription processes that are important for the progression, invasion and growth of metastatic epithelial cells $(25,26)$. In colorectal cancer, as a regulator of tumor-associated lymphangiogenesis, MTA1 promotes lymphangiogenesis by mediating the expression of vascular endothelial growth factor (VEGF)-C (27).

The expression of metastasis suppressor gene nm23 was shown to be higher in neoplastic colorectal carcinoma tissue compared to the adjacent non-neoplastic mucosa, exhibiting a correlation with longer disease-free survival (28). The overexpression of nm23 may be involved in carcinogenesis and a decrease in nm23 expression was previously shown to be associated with metastasis of GI carcinoma (29).

The KISS-1 gene maps to chromosome 1 bands q32-q41 and encodes a largely hydrophobic 145 -amino acid protein. KISS-1 acts as a tumor metastasis suppressor and its expression is weak or undetectable in human tumors. In gastric carcinoma, KISS-1 may inhibit the invasion and metastasis in vitro and in vivo, through the downregulation of matrix metalloproteinase (MMP)-9 (30). Aberrant expression of KISS-1 and MMP-9 are closely associated with lymph node metastasis (31).

KAI1 (CD82), another tumor metastasis suppressor gene, belongs to the transmembrane 4 superfamily, the members of which exert inhibitory effects on tumor cell motility and metastasis. The loss of KAI1 may be considered a significant prognostic parameter in the prediction of the progression of GI carcinomas, when evaluated along with clinical and pathological parameters $(32,33)$. Spliced KAI1, in which exon 7 has been deleted, differs from the wild-type KAI1 with respect to cell motility, adhesion, tumor growth and metastasis. The expression of spliced KAI1 may be a better marker for poor prognosis in gastric cancer patients (34).

Other metastasis-associated genes, such as girdin, chemokine ligand (CCL)2 and SNCG, may be used as accurate predictors of liver metastasis in colorectal cancer when combined with clinicopathological characteristics $(35,36)$. NR4A2 and HSP90AA1 have been considered promising diagnostic markers and therapeutic targets for the liver metastasis of gastric cancer (37). The S100A4 and CTNNB1 genes 
were also closely associated with peritoneal metastasis of gastric adenocarcinoma (38).

\section{Cell cycle and apoptosis regulators}

Aberrations in the cell cycle regulators are common in several tumors and have been shown to be of prognostic significance in GI carcinoma. Cyclin and cyclin-dependent kinase (CDK) are crucial in cell cycle regulation. The overexpression of cyclin E and CDK-2 and the downregulated expression of p57 (KIP2) may play important roles in the development and metastatic potential of gastric cancer (39). A previous study demonstrated that there was no significant association between cyclin E expression and clinicopathological variables; however, cyclin G2 appeared to be a negative cell cycle regulator in gastric cancer, with its expression being inversely correlated with gastric cancer progression (40). In addition, the expression of cyclin $\mathrm{E}$ was not identified as a predictor of survival in a series of patients with gastric cancer (41). Therefore, cyclin E may not be a suitable biomarker. Cyclin D1 overexpression was associated with longer survival in colon cancer patients (42) and the coexistence of cyclin D1 and VEGF protein expression was shown to be a poor prognostic factor for UICC stage I-III colorectal cancer patients following curative resection (43). Of note, cyclin D1 expression was significantly associated with prolonged survival in male colorectal cancer patients. Those findings supported an important role for cyclin D1 in colorectal carcinogenesis and added further weight to the accumulated evidence indicating that colorectal cancer was indeed a hormone-dependent malignancy, for which prognostic and treatment-predictive molecular biomarkers should be differentially evaluated in women and men (44). The expression of cyclin D1 and P21WAF1 was also associated with tumor drug resistance. Therefore, the measurement of cyclin D1 and P21WAF1 may be of reference value in the selection of chemotherapeutic drugs (45).

Cyclin L2 is a novel member of the cyclin family. The overexpression of the cyclin L2 protein may promote efficient growth inhibition and enhance chemosensitivity to chemotherapeutic agents in human gastric cancer cells through the induction of G0/G1 cell cycle arrest and apoptosis (46). CDK8, the gene encoding the CDK component of the mediator complex, may lead to the identification of a subset of colon cancer patients with a poor prognosis (47).

The p16 gene is crucial for cell cycle regulation. The expression of p16, alone or in combination with p53, was shown to be predictive of a better prognosis in colorectal adenocarcinoma in Tunisian patients (48). The repression of p16 may promote cell growth and metastasis in colorectal cancer (46). In addition, p16 methylation in the serum may be used as a follow-up marker for the recurrence of colorectal cancer (50).

The expression of cell cycle regulators p21 and p27 has been used as a predictor of disease outcome in colorectal carcinoma (51). Previously reported data suggested that CDK8 detection and $\beta$-catenin delocalization may be associated with a poor prognosis and, thus, CDK8 interference may be a promising therapeutic modality for gastric adenocarcinoma (52).

CDK subunit 1 (Cks1) and S-phase kinase-associated protein 2 (Skp2) were shown to regulate the expression level of the p27 protein and play an important role in gastric carcinoma progression, which renders them a novel target for the treatment of gastric carcinoma, as well as strong prognostic markers (53). CDKL1 may also be a novel therapeutic target (54).

Apoptosis, or programmed cell death, via the caspase cascade, is involved in tumorigenesis and cancer progression. Thus, the altered expression of apoptosis-related proteins may be of clinical and prognostic significance. Moreover, the apoptotic pathway is highlighted due to the recent introduction of apoptosis-targeted therapy for several genes. The p53 protein, as well as the Bcl-2 family proteins, such as Bax, Bak and $\mathrm{Bcl}-\mathrm{xL}$ may regulate apoptosis. Disturbances in the expression of the p53, Bax, Bcl-xL and Bak proteins have been associated with their involvement in the process of gastric carcinogenesis and it was suggested that they may appear during the early phases of carcinogenesis (55). The efficacy of adjuvant chemotherapy for gastric cancer may be affected by the status of apoptosis-related proteins, such as p53, p21, Bax and Mcl-1 (56). The expression of Bax was shown to be predictive of a favorable clinical outcome in chemonaive patients with advanced gastric cancer treated with capecitabine, oxaliplatin and irinotecan (57). A low expression of Bax was significantly associated with poor survival of patients with metastatic or recurrent gastric cancer treated with the FOLFOX regimen. The immunohistochemical staining for Bax in pretreatment biopsy specimens may be useful in the selection of the FOLFOX regimen as a treatment option for patients with advanced gastric cancer (58). The expression of Bcl-2 was identified as an independent prognostic factor for Chinese patients with gastric cancer and it may be a candidate for the gastric cancer staging system (59). The patient group that expressed Bcl-2 exhibited longer survival (60). The regulation of the $\mathrm{Bax} / \mathrm{Bcl}-2$ ratio and the caspase-9-dependent pathway may induce apoptosis (61). Moreover, the expression of the $\mathrm{X}$-linked inhibitor of apoptosis protein (XIAP) was associated with a poor prognosis and the expression of cleaved caspase-3 was associated with early cancer stage and they were both identified as independent prognostic factors. Those findings may help identify gastric cancer patients that are likely to benefit from apoptosis-targeted therapy (62).

\section{Cell adhesion molecules}

Cell adhesion molecules (CAMs) play a vital role in the metastatic potential of GI carcinoma and, thus, affect the prognosis of this type of malignancy. The downregulation of cadherins and catenins facilitates tumor cell detachment from the primary site, whereas the expression of integrins, selectins and members of the immunoglobulin superfamily may support neoplastic progression, intravasation and malignant cell attachment to foreign tissue, leading to the development of metastases (63). The classical cadherins comprise E-, P-, N- and R-cadherin. Establishing the stability of the cadherin-catenin complex significantly contributes to cell-cell adhesive junctions in epithelial tissues, playing pivotal roles in important morphogenetic and differentiation processes during development and in maintaining integrity and homeostasis in adult tissues. In general, cadherin genes are regarded as tumor suppressors. Currently, it is widely accepted that downregulation of these molecules is observed during tumor progression and often results in tissue disorder, cellular de-differentiation, 
increased invasiveness of tumor cells and, ultimately, metastasis (64). The detection of E-cadherin expression may help characterize gastric carcinoma histotypes $(65,66)$ and the loss of E-cadherin may promote tumor growth, invasion and drug resistance and be associated with metastasis (64). Therefore, E-cadherin may prove useful in predicting prognosis and designing adjuvant therapy in GI carcinoma patients (68). Measuring serum E-cadherin levels may also provide valuable information (69). In addition, elevated $\mathrm{N}$ - and P-cadherin expression may be a useful marker independent of E-cadherin expression, favoring a better prognosis $(70,71)$. However, it was recently demonstrated that P-cadherin contributes to the development of several types of tumors, including gastric and colon carcinomas, which suggests that blocking the activity of P-cadherin or its associated signaling pathway may be a valuable treatment target $(72,73)$. Therefore, further investigation of the function of P-cadherin is required.

Accumulating evidence indicates that selectins in conjunction with their ligands may regulate circulating cancer cell communication and adhesion to the walls of blood vessels (74). Recent clinical studies investigated the role of selectins in GI carcinoma and measurements of their soluble forms demonstrated that high serum values were significantly correlated with metastasis, prognosis and relapse $(75,76)$.

Integrins are heterodimer cell surface receptors that interact with the extracellular matrix (ECM) and regulate intracellular communication via outside-in and inside-out signaling. Functional blocking of specific integrins may inhibit GI carcinoma invasion and migration (77).

The immunoglobulin superfamily consists of numerous cell surface and soluble proteins, such as intercellular adhesion molecules (ICAMs) and vascular cell adhesion molecule 1 (VCAM-1), which are involved in intercellular recognition, binding and adhesion. The increased serum concentrations of ICAM-1 and VCAM-1 were highly associated with metastasis and recurrence in GI carcinoma and, thus, may prove to be useful clinical biomarkers $(78,79)$.

The involvement of CAMs in numerous aspects of GI carcinoma progression, distant metastases and relapse has been applied in therapeutic experiments and clinical trials. Anti-adhesion treatment against GI carcinoma is currently under development and may be successfully used in the clinical practice in the immediate future (63).

\section{Growth factors, cytokines and angiogenic factors}

The tumor microenvironment plays a vital role in tumor proliferation, invasion and metastasis and GI carcinoma is no exception. Numerous cytokines and growth factors are an important component of the tumor microenvironment. It was previously determined that human GI carcinoma overexpresses numerous cytokines, growth factors and their receptors, such as tumor necrosis factor (TNF), transforming growth factor (TGF), interleukin (IL), chemotactic cytokines (chemokines), VEGF, fibroblast growth factor (FGF), epidermal growth factor (EGF) family and insulin-like growth factor (IGF). The abundance of growth-promoting signaling and the disturbance of growth inhibitory signaling may lead to self-sufficiency in growth signaling and evasion of programmed cell death and to enhancement of angiogen- esis, tumor growth and metastasis (6). The investigation and analysis of the expression of cytokines, growth factors and their receptors may be associated with the clinical diagnosis, treatment and prognosis of GI carcinoma.

In the tumor microenvironment, IL- 8 and its chemokine receptor 2 (CXCR2) promoted gastric (80) and colon cancer growth, progression and metastasis $(81,82)$. The expression of IL-4, IL-4R and IL-13R were involved in the process of local metastasis of colorectal cancer, whereas the expression of IL-13 has been shown to affect survival. These interleukins and their receptors may prove to be attractive targets for the treatment of colorectal cancer (83). In addition, the detection of IL-4, IL-6 and IL-10 expression may be useful as a molecular marker approach for distinguishing stages II and III of gastric cancer, as well as low-level and moderate cancer differentiation (84).

The FGF receptor (FGFR) family and their ligands, FGFs, are differentially expressed in GI carcinoma. It was previously suggested that FGF receptor signaling may contribute to the progression of GI carcinoma and a monoclonal antibody against FGFR appears promising in clinical therapy $(85,86)$.

Chemokine CCL7 and CCL21 overexpression in gastric cancer was associated with lymph node metastasis and poor prognosis (87) and CCL5 was involved in the pathogenesis of colorectal carcinoma (88). However, CCL25 and its cognate receptor CXCR9 inhibit colorectal cancer invasion and metastasis (89). The stromal cell-derived factor-1 (SDF-1) and its receptor CXCR4 play pivotal roles in tumor metastasis and drug resistance. Thus, hampering the SDF-1/CXCR4 crosstalk may be a promising strategy for clinical therapy $(90,91)$.

It has been established that TGF- $\beta 1$ signaling is crucial in connecting aberrant inflammation to colorectal tumorigenesis (92). Data on TGF- $\beta 1$ and TGF- $\beta$ RII expression demonstrated an association with clinicopathological parameters, particularly differentiation and histological type. It was previously reported that TGF- $\beta 1$-positive patients exhibited a shorter overall survival compared to TGF- $\beta 1$-negative patients with gastric cancer (93). Furthermore, EGFR positivity may be associated with poor patient outcomes following curative resection of stage II/III gastric cancer (94).

The hepatocyte growth factor (HGF)/c-Met signaling pathway is involved in the pathogenesis and progression of colon cancer (95). Solid tumor growth is dependent on angiogenesis, which provides oxygen and nutrients to the metastatic site and facilitates the development of metastasis.

During tumor angiogenesis, the critical steps are the outgrowth of endothelial cells from pre-existing capillary vessels and their migration from parental vessels (96) and VEGF is considered a prime mediator of this process. VEGF receptor (VEGFR)-2 and VEGFR-3 expression were identified as markers of poor prognosis in patients with surgically resected colorectal adenocarcinoma (97). Overexpression of VEGF was shown to be an important predictor of early postoperative relapse (98) and it was suggested that the inhibition of VEGF may promote the senescence of colorectal cancer cells (99). Accumulating evidence indicates that the clinical outcome may be associated with VEGF overexpression in non-tumor areas $\left(\mathrm{VEGF}_{\mathrm{nt}}\right)$. VEGF expression in colorectal cancer tissue was shown to be higher in tumor $\left(\mathrm{VEGF}_{t}\right)$ 
compared to that in non-tumor areas. Indeed, patients with a $\mathrm{VEGF}_{\mathrm{t}} / \mathrm{VEGF}_{\mathrm{nt}}$ ratio of $>2$ exhibited longer survival (100). It was also reported that VEGF small interfering RNA may induce apoptosis (101). In addition, serum VEGF tracking may be useful in monitoring tumor progression. Preoperative measurement of serum VEGF levels may help identify patients with a poor prognosis $(102,103)$. Furthermore, VEGF gene polymorphisms may be associated with gastric cancer in Chinese Han patients and the differences in genotype distribution may be associated with the location and Lauren's classification of gastric cancer (104).

\section{Matrix metalloproteinases (MMPs)}

The controlled degradation of the ECM is essential for the growth, invasion and metastasis of malignant tumors and for tumor-induced angiogenesis. MMPs are a family of zinc-dependent neutral endopeptidases, collectively capable of degrading the ECM components and they appear to play an important role in all the aspects of tumor development (105). A positive correlation was previously demonstrated between MMP expression and the invasive and metastatic potential of GI carcinoma. High levels of MMP-1 mRNA expression were detected in the peripheral blood and bone marrow of gastric cancer patients, accurately reflecting gastric cancer stage. Therefore, MMP-1 may be involved in the development and metastasis of gastric cancer (106). As an independent prognostic factor of colon carcinoma, the expression of MMP-1 in cancer tissue exhibits different prognostic implications according to various stages (107).

MMP-2 was shown to be an important factor associated with the development and metastasis of gastric and colorectal cancer and may be used as a prognostic marker $(108,109)$.

The overexpression of MMP-7 may play an important role in the pathogenesis of colorectal cancer and the combined detection with a reduced PTEN expression may provide prognostic benefit for colorectal cancer $(110,111)$. Furthermore, MMP-7 was associated with poor overall survival in gastric cancer (112).

Other MMPs, such as MMP-15 and -19, were identified as stromal regulators in the early stages of colorectal cancer development and may also prove to be useful biomarkers for GI carcinoma (113).

The inhibition of MMPs has been investigated as a therapeutic target. Under normal physiological conditions, MMP activity is regulated by the tissue inhibitors of metalloproteinases (TIMPs) and any alteration in this regulatory process may lead to pathological conditions. Although previous studies demonstrated the expression of TIMPs in the tumor tissue and stroma, the prognostic value of TIMP expression has not established. For example, the overexpression of TIMP-1 may correlate with poor outcomes in gastric and colorectal cancer $(114,115)$, although it was also reported that TIMP-1 expression in gastric cancer cells may prevent metastasis (116). Similarly, the transfection of the TIMP-1 gene through adenoviral mediation exerted a suppressive effect on peritoneal metastases from gastric cancer (117). The imbalance between MMPs and TIMPs may facilitate the progression and metastasis of cancer cells. The evaluation of either MMP or TIMP expression alone may not always be sufficient for prognostica- tion of malignancies, with the MMP:TIMP ratio being more descriptive (118). Therefore, the estimation and adjustment of the MMP:TIMP ratio may become a clinical diagnostic and therapeutic target in the future.

\section{MicroRNAs}

MicroRNAs (miRNAs) are small, non-coding RNA segments that regulate gene expression at the post-transcriptional level and are crucial in cancer development, progression and metastasis. Specific miRNAs may act as either tumor suppressors or oncogenes, depending on the cellular environment within which they are expressed. The significant biological stability of miRNAs opens novel fields in biomarker research, with potential clinical implementation in screening, diagnosis, prediction of prognosis and therapeutic management. There is an increasing understanding of the potential use of miRNAs as biomarkers of GI carcinoma. Plasma miRNAs, such as miR-223, -21, -218, -199a-3p, -221, -376c and -744, constitute reliable and non-invasive markers and show potential as novel biomarkers for the early detection of gastric cancer $(119,120)$. In addition, gastric juice miRNAs, such as miR-421, were shown to be useful biomarkers (121). Circulating miR-17-5p/20a and miR-200c may be promising molecular markers for the pathological progression, prediction of prognosis and monitoring of the chemotherapeutic effects of gastric cancer $(122,123)$. Increased plasma miR-21 levels were detected in colorectal cancer, whereas circulating miR-34a levels were reduced, representing a promising biomarker for colorectal cancer $(124,125)$. In addition, tissue miRNAs, such as miR-107 and -223, were shown to act as oncogenes in human gastric cancer, promoting cancer invasion and metastasis $(126,127)$. miR-21, $-31,-96,-135 b$ and -92 a may be involved in colorectal cancer development and progression and miR-135b levels, in particular, may correlate with the degree of malignancy $(128,129)$. miR-200c was shown to play an important role in mediating epithelial-mesenchymal transition (EMT) and metastatic behavior in colon cancer. Its expression is epigenetically regulated and miR-200c may serve as a potential diagnostic marker and therapeutic target for patients with colorectal cancer (130). According to previous studies, miR-155, -495 and -551a may act as tumor suppressors to regulate gastric cancer cell invasion and metastasis and those findings contributed to the understanding of the functions of miRNA mimics in gastric cancer gene therapy $(131,132)$. In patients harboring KRAS mutations, let-7a analysis may help identify subgroups of patients who may benefit from EGFR inhibition, opening up new perspectives for alternative treatment strategies (133). It was demonstrated that miR-222 modulated multidrug resistance (MDR) in human colorectal carcinoma by downregulating ADAM metallopeptidase domain 17 (ADAM-17), suggesting that miR-222 plays a role in the development of MDR through the modulation of ADAM-17, a novel MDR treatment target in colorectal carcinoma cells (134). Furthermore, miRNA profiling using formalin-fixed, paraffin-embedded samples may be a useful and promising evaluation method of samples stored in laboratories worldwide and may generate valuable clinical data in gastric cancer patients, identifying miR-34a as an independent prognostic factor (135). 


\section{Conclusion}

The development of GI carcinoma is a complex, multistep process, involving numerous genetic and epigenetic alterations in oncogenes, tumor suppressor genes, DNA repair genes, telomeres and telomerases, cell cycle regulators and signaling molecules. Genomic instability, either microsatellite or chromosomal, leads to the accumulation of genetic alterations and participates in the pathogenetic process. Additionally, certain aberrant epigenetic modifications are closely associated with tumor progression and may be candidate diagnostic and prognostic factors. Among the various epigenetic alterations that lead to modified gene expression, the most important are considered to be DNA methylation and chromatin remodeling by histone modification. Furthermore, genetic polymorphism is an important determinant of the endogenous causes of cancer.

An important objective of current investigations is to identify novel biomarkers that may detect tumors in the early stages and accurately indicate the status of the disease. The availability of validated biological markers for early tumor detection, selection of adjuvant therapy, prediction and monitoring of treatment efficacy are likely to increase survival. The aim of this review was to summarize the potential biomarkers for GI carcinoma. Any molecular alterations, genetic and/or epigenetic, involved in the progression and metastasis of GI carcinoma, may be identified as a valuable biomarker. Currently, with the development of the 'omic' and other new technologies, it is easier to achieve a comprehensive approach to cancer biomarker identification. A limitation of the present review is that we only addressed the expression of well-known genes or proteins, such as TAAs, oncogenes and tumor suppressor genes, metastasis-associated genes, cytokines, growth factors and miRNAs. In addition, due to the numerous molecular alterations and the complications in the histopathology of GI carcinoma, the significance of these molecular changes for the pathogenesis of GI carcinoma remains to be further elucidated. The identification and characterization of the critical molecular alterations in GI carcinoma may hold great promise for the screening, classification, diagnosis, prognosis and, most importantly, the development of novel therapeutic targets for this type of malignancy.

\section{Acknowledgements}

This study was supported by the National Natural Science Foundation of China (grant no. 81071667).

\section{References}

1. Ferlay J, Shin HR, Bray F, Forman D, Mathers C and Parkin DM: Estimates of worldwide burden of cancer in 2008: GLOBOCAN 2008. Int J Cancer 127: 2893-2917, 2010.

2. Jemal A, Bray F, Center MM, Ferlay J, Ward E and Forman D: Global cancer statistics. CA Cancer J Clin 61: 69-90, 2011.

3. Ebert MP, Schandl L and Malfertheiner P: Helicobacter pylori infection and molecular changes in gastric carcinogenesis. J Gastroenterol 37 (Suppl 13): 45-49, 2002.

4. Krejs GJ: Gastric cancer: epidemiology and risk factors. Dig Dis 28: 600-603, 2010.

5. Nagini S: Carcinoma of the stomach: A review of epidemiology, pathogenesis, molecular genetics and chemoprevention. World $\mathbf{J}$ Gastrointest Oncol 4: 156-169, 2012.
6. Zheng L, Wang L, Ajani J and Xie K: Molecular basis of gastric cancer development and progression. Gastric Cancer 7: 61-77, 2004.

7. Remo A, Pancione M,Zanella C and Vendraminelli R: Molecular pathology of colorectal carcinoma. A systemic review centred on the new role of the pathologist. Pathologica 104: 432-441, 2012.

8. Zayakin P, Ancans G, Silina K, et al: Tumor-associated autoantibody signature for the early detection of gastric cancer. Int $\mathrm{J}$ Cancer 132: 137-147, 2013.

9. Lu H, Goodell V and Disis ML: Humoral immunity directed against tumor-associated antigens as potential biomarkers for the early diagnosis of cancer. J Proteome Res 7: 1388-1394, 2008.

10. Grotowski M: Antigens (CEA and CA 19-9) in diagnosis and prognosis colorectal cancer. Pol Merkur Lekarski 12: 77-80, 2002 (In Polish).

11. Takahashi Y: Gastrointestinal cancer. Gan To Kagaku Ryoho 31: 1275-1279, 2004 (In Japanese).

12. Park IJ, Choi GS and Jun SH: Prognostic value of serum tumor antigen CA19-9 after curative resection of colorectal cancer. Anticancer Res 29: 4303-4308, 2009.

13. Fernandes LL, Martins LC, Nagashima CA, Nagae AC, Waisberg DR and Waisberg J: CA72-4 antigen levels in serum and peritoneal washing in gastric cancer. Correlation with morphological aspects of neoplasia. Arq Gastroenterol 44: 235-239, 2007.

14. Carpelan-Holmstrom M, Louhimo J, Stenman UH, Alfthan $H$ and Haglund C: CEA, CA 19-9 and CA 72-4 improve the diagnostic accuracy in gastrointestinal cancers. Anticancer Res 22: 2311-2316, 2002

15. Chen XZ, Zhang WK, Yang K, et al: Correlation between serum CA724 and gastric cancer: multiple analyses based on Chinese population. Mol Biol Rep 39: 9031-9039, 2012.

16. Van Cutsem E, Kohne CH, Lang I, et al: Cetuximab plus irinotecan, fluorouracil, and leucovorin as first-line treatment for metastatic colorectal cancer: updated analysis of overall survival according to tumor KRAS and BRAF mutation status. J Clin Oncol 29: 2011-2019, 2011.

17. Joyce T, Oikonomou E, Kosmidou V, et al: A molecular signature for oncogenic BRAF in human colon cancer cells is revealed by microarray analysis. Curr Cancer Drug Targets 12: 873-898, 2012.

18. Lee J, Seo JW, Jun HJ, et al: Impact of MET amplification on gastric cancer: Possible roles as a novel prognostic marker and a potential therapeutic target. Oncol Rep 25: 1517-1524, 2011.

19. Bayrak M, Olmez OF, Kurt E, et al: Prognostic significance of c-erbB2 overexpression in patients with metastatic gastric cancer. Clin Transl Oncol 15: 307-312, 2013.

20. Zhong J, Zhao M, Ma Y, et al: UCHL1 acts as a colorectal cancer oncogene via activation of the $\beta$-catenin/TCF pathway through its deubiquitinating activity. Int J Mol Med 30: 430-436, 2012.

21. Bellini MF, Cadamuro AC, Succi M, Proenca MA and Silva AE: Alterations of the TP53 gene in gastric and esophageal carcinogenesis. J Biomed Biotechnol 2012: 891961, 2012.

22. Fang Z, Xiong Y, Li J, et al: APC gene deletions in gastric adenocarcinomas in a Chinese population: a correlation with tumour progression. Clin Transl Oncol 14: 60-65, 2012.

23. Hsu CP, Kao TY, Chang WL, Nieh S, Wang HL and Chung YC: Clinical significance of tumor suppressor PTEN in colorectal carcinoma. Eur J Surg Oncol 37: 140-147, 2011.

24. Tate G, Suzuki T, Nemoto H, Kishimoto K, Hibi K and Mitsuya T: Allelic loss of the PTEN gene and mutation of the TP53 gene in choriocarcinoma arising from gastric adenocarcinoma: analysis of loss of heterozygosity in two male patients with extragonadal choriocarcinoma. Cancer Genet Cytogenet 193: 104-108, 2009.

25. Nicolson GL, Nawa A, Toh Y, Taniguchi S, Nishimori K and Moustafa A: Tumor metastasis-associated human MTA1 gene and its MTA1 protein product: role in epithelial cancer cell invasion, proliferation and nuclear regulation. Clin Exp Metastasis 20: 19-24, 2003.

26. Toh Y, Oki E, Oda S, et al: Overexpression of the MTA1 gene in gastrointestinal carcinomas: correlation with invasion and metastasis. Int J Cancer 74: 459-463, 1997.

27. Du B, Yang ZY, Zhong XY, et al: Metastasis-associated protein induces VEGF-C and facilitates lymphangiogenesis in colorectal cancer. World J Gastroenterol 17: 1219-1226, 2011.

28. Oliveira LA, Artigiani-Neto R, Waisberg DR, Fernandes LC, Lima Fde O and Waisberg J: NM23 protein expression in colorectal carcinoma using TMA (tissue microarray): association with metastases and survival. Arq Gastroenterol 47: 361-367, 2010 . 
29. Tahara E, Kuniyasu H, Nakayama H, Yasui W and Yokozaki H: Metastasis related genes and malignancy in human esophageal, gastric and colorectal cancers. Gan To Kagaku Ryoho 20 326-331, 1993 (In Japanese).

30. Li N, Wang HX, Zhang J, Ye YP and He GY: KISS-1 inhibits the proliferation and invasion of gastric carcinoma cells. World J Gastroenterol 18: 1827-1833, 2012

31. Zheng HC, Yu AM and Xin Y: Aberrant expression of Kiss-1 and matrix metalloproteinase- 9 are closely linked to lymph node metastasis of gastric cancer. Chin Med Sci J 23: 63-64, 2008

32. Ilhan O, Celik SY, Han U and Onal B: Use of KAI-1 as a prognostic factor in gastric carcinoma. Eur J Gastroenterol Hepatol 21: 1369-1372, 2009.

33. Lombardi DP, Geradts J, Foley JF, Chiao C, Lamb PW and Barrett JC: Loss of KAI1 expression in the progression of colorectal cancer. Cancer Res 59: 5724-5731, 1999.

34. Lee JH, Seo YW, Park SR, Kim YJ and Kim KK: Expression of a splice variant of KAI1, a tumor metastasis suppressor gene, influences tumor invasion and progression. Cancer Res 63: 7247-7255, 2003

35. Hu H, Sun L, Guo C, et al: Tumor cell-microenvironment interaction models coupled with clinical validation reveal CCL2 and SNCG as two predictors of colorectal cancer hepatic metastasis. Clin Cancer Res 15: 5485-5493, 2009

36. Liu C, Xue H, Lu Y and Chi B: Stem cell gene Girdin: a potential early liver metastasis predictor of colorectal cancer. Mol Bio Rep 39: 8717-8722, 2012

37. Chang W, Ma L, Lin L, et al: Identification of novel hub genes associated with liver metastasis of gastric cancer. Int J Cancer 125: 2844-2853, 2009.

38. Bai FH, Wang NJ, Wang J, et al: Screening and identification of peritoneal metastasis-related genes of gastric adenocarcinoma using a cDNA microarray. Genet Mol Res 11: 1682-1689, 2012.

39. Liang B, Wang S, Yang X, Ye Y, Yu Y and Cui Z: Expressions of cyclin E, cyclin dependent kinase 2 and p57(KIP2) in human gastric cancer. Chin Med J (Engl) 116: 20-23, 2003.

40. Choi MG, Noh JH, An JY, et al: Expression levels of cyclin G2, but not cyclin E, correlate with gastric cancer progression. J Surg Res 157: 168-174, 2009

41. Kouraklis G, Katsoulis IE, Theocharis S, et al: Does the expression of cyclin $\mathrm{E}, \mathrm{pRb}$, and $\mathrm{p} 21$ correlate with prognosis in gastric adenocarcinoma? Dig Dis Sci 54: 1015-1020, 2009.

42. Ogino S, Nosho K, Irahara N, et al: A cohort study of cyclin D1 expression and prognosis in 602 colon cancer cases. Clin Cancer Res 15: 4431-4438, 2009.

43. Tsai HL, Yeh YS, Chang YT, et al: Co-existence of cyclin D1 and vascular endothelial growth factor protein expression is a poor prognostic factor for UICC stage I-III colorectal cancer patients after curative resection. J Surg Oncol 107: 148-154, 2013.

44. Wangefjord S, Manjer J, Gaber A, Nodin B, Eberhard J and Jirstrom K: Cyclin D1 expression in colorectal cancer is a favorable prognostic factor in men but not in women in a prospective, population-based cohort study. Biol Sex Differ 2 . 10, 2011.

45. Geng M, Wang L, Cao YC, Li H and Liu XH: Relationship of the expression of cyclin D1 and P21WAF1 with the sensitivity to chemotherapeutic drugs on gastric cancers. Chin J Gastroenterol Surg 12: 615-617, 2009 (In Chinese).

46. Li HL, Huang DZ, Deng T, et al: Overexpression of cyclin L2 inhibits growth and enhances chemosensitivity in human gastric cancer cells. Asian Pac J Cancer Prev 13: 1425-1430, 2012.

47. Firestein R, Shima K, Nosho K, et al: CDK8 expression in 470 colorectal cancers in relation to $\beta$-catenin activation, other molecular alterations and patient survival. Int J Cancer 126 : 2863-2873, 2010.

48. Miladi-Abdennadher I, Abdelmaksoud-Damak R, Ayadi L, et al: Expression of p16INK4a, alone or combined with p53, is predictive of better prognosis in colorectal adenocarcinoma in Tunisian patients. Appl Immunohistochem Mol Morphol 19: 562-568, 2011

49. Wang F, Yang YZ, Shi CZ, et al: UHRF1 promotes cell growth and metastasis through repression of p16 $6^{\text {inka }}$ in colorectal cancer. Ann Surg Oncol 19: 2753-2762, 2012.

50. Nakayama G, Kodera Y, Ohashi N, Koike M, Fujiwara M and Nakao A: p16 ${ }^{\text {INK4a }}$ methylation in serum as a follow-up marker for recurrence of colorectal cancer. Anticancer Res 31: 1643-1646, 2011.

51. Al-Maghrabi J, Al-Ahwal M, Buhmeida A, et al: Expression of cell cycle regulators $\mathrm{p} 21$ and $\mathrm{p} 27$ as predictors of disease outcome in colorectal carcinoma. J Gastrointest Cancer 43: 279-287, 2012.
52. Kim MY, Han SI and Lim SC: Roles of cyclin-dependent kinase 8 and $\beta$-catenin in the oncogenesis and progression of gastric adenocarcinoma. Int J Oncol 38: 1375-1383, 2011.

53. Masuda TA, Inoue H, Nishida K, et al: Cyclin-dependent kinase 1 gene expression is associated with poor prognosis in gastric carcinoma. Clin Cancer Res 9: 5693-5698, 2003.

54. Sun W, Yao L, Jiang B, Shao H, Zhao Y and Wang Q: A role for Cdk11 in the development of gastric cancer. Acta Oncol 51: 790-796, 2012

55. Gryko M, Pryczynicz A, Guzinska-Ustymowicz K, et al: Immunohistochemical assessment of apoptosis-associated proteins: p53, Bcl-xL, Bax and Bak in gastric cancer cells in correlation with clinical and pathomorphological factors. Adv Med Sci 57: 77-83, 2012.

56. Tsujitani S, Saito H, Wakatsuki T, et al: Relationship between expression of apoptosis-related proteins and the efficacy of postoperative chemotherapy in patients with T3 gastric cancer. Surg Today 42: 225-232, 2012

57. Pietrantonio F, Biondani $\mathrm{P}$, de Braud F, et al: Bax expression is predictive of favorable clinical outcome in chemonaive advanced gastric cancer patients treated with capecitabine, oxaliplatin, and irinotecan regimen. Transl Oncol 5: 155-159, 2012.

58. Jeong $\mathrm{SH}, \mathrm{Han} \mathrm{JH}, \mathrm{Kim} \mathrm{JH}$, et al: Bax predicts outcome in gastric cancer patients treated with 5-fluorouracil, leucovorin, and oxaliplatin palliative chemotherapy. Dig Dis Sci 56: 131-138, 2011.

59. Liu X, Cai H, Huang H, Long Z, Shi Y and Wang Y: The prognostic significance of apoptosis-related biological markers in Chinese gastric cancer patients. PLoS One 6: e29670, 2011.

60. Yildirim M, Suren D, Goktas S, et al: The predictive role of Bcl-2 expression in operable locally advanced or metastatic gastric carcinoma. J BUON 17: 106-109, 2012.

61. Lee JS, Jung WK, Jeong MH, Yoon TR and Kim HK: Sanguinarine induces apoptosis of HT-29 human colon cancer cells via the regulation of $\mathrm{Bax} / \mathrm{Bcl}-2$ ratio and caspase-9-dependent pathway. Int J Toxicol 31: 70-77, 2012.

62. Kim MA, Lee HE, Lee HS, Yang HK and Kim WH: Expression of apoptosis-related proteins and its clinical implication in surgically resected gastric carcinoma. Virchows Arch 459: 503-510, 2011.

63. Paschos KA, Canovas D and Bird NC: The role of cell adhesion molecules in the progression of colorectal cancer and the development of liver metastasis. Cell Signal 21: 665-674, 2009.

64. Paredes J, Figueiredo J, Albergaria A, et al: Epithelial E- and P-cadherins: role and clinical significance in cancer. Biochim Biophys Acta 1826: 297-311, 2012.

65. Almeida PR, Ferreira VA, Santos CC, et al: E-cadherin immunoexpression patterns in the characterisation of gastric carcinoma histotypes. J Clin Pathol 63: 635-639, 2010.

66. Stanculescu D, Margaritescu C, Stepan A and Mitrut AO: E-cadherin in gastric carcinomas related to histological prognostic parameters. Rom J Morphol Embryol 52 (Suppl 3): 1107-1112, 2011.

67. Chen X, Wang Y, Xia H, et al: Loss of E-cadherin promotes the growth, invasion and drug resistance of colorectal cancer cells and is associated with liver metastasis. Mol Biol Rep 39: 6707-6714, 2012

68. Kang H, Min BS, Lee KY, et al: Loss of E-cadherin and MUC2 expressions correlated with poor survival in patients with stages II and III colorectal carcinoma. Ann Surg Oncol 18: 711-719, 2011.

69. Okugawa Y, Toiyama Y, Inoue Y, et al: Clinical significance of serum soluble E-cadherin in colorectal carcinoma. J Surg Res 175: e67-e73, 2012.

70. Kamikihara T, Ishigami S, Arigami T, et al: Clinical implications of $\mathrm{N}$-cadherin expression in gastric cancer. Pathol Int 62: 161-166, 2012.

71. Kim MA, Jung EJ, Lee HS, et al: P-cadherin expression in gastric carcinoma: its regulation mechanism and prognostic significance. Hum Pathol 41: 877-885, 2010.

72. Sun L, Hu H, Peng L, et al: P-cadherin promotes liver metastasis and is associated with poor prognosis in colon cancer. Am J Pathol 179: 380-390, 2011.

73. Park J, Park E, Han SW, et al: Down-regulation of P-cadherin with PF-03732010 inhibits cell migration and tumor growth in gastric cancer. Invest New Drugs 30: 1404-1412, 2012.

74. St Hill CA: Interactions between endothelial selectins and cancer cells regulate metastasis. Front Biosci 16: 3233-3251, 2011.

75. Ferroni P, Roselli M, Spila A, et al: Serum sE-selectin levels and carcinoembryonic antigen mRNA-expressing cells in peripheral blood as prognostic factors in colorectal cancer patients. Cancer 116: 2913-2921, 2010 
76. Sato H, Usuda N, Kuroda M, Hashimoto S, Maruta M and Maeda K: Significance of serum concentrations of E-selectin and CA19-9 in the prognosis of colorectal cancer. Jpn J Clin Oncol 40: 1073-1080, 2010.

77. Robertson JH, Yang SY, Winslet MC and Seifalian AM: Functional blocking of specific integrins inhibit colonic cancer migration. Clin Exp Metastasis 26: 769-780, 2009.

78. Alexiou D, Karayiannakis AJ, Syrigos KN, et al: Clinical significance of serum levels of E-selectin, intercellular adhesion molecule-1, and vascular cell adhesion molecule-1 in gastric cancer patients. Am J Gastroenterol 98: 478-485, 2003.

79. Dymicka-Piekarska V, Guzinska-Ustymowicz K, Kuklinski A and Kemona $\mathrm{H}$ : Prognostic significance of adhesion molecules (sICAM-1, sVCAM-1) and VEGF in colorectal cancer patients. Thromb Res 129: e47-e50, 2012.

80. Kuai WX, Wang Q, Yang XZ, Zhao Y, Yu R and Tang XJ: Interleukin-8 associates with adhesion, migration, invasion and chemosensitivity of human gastric cancer cells. World J Gastroenterol 18: 979-985, 2012

81. Lee YS, Choi I, Ning Y, et al: Interleukin-8 and its receptor CXCR2 in the tumour microenvironment promote colon cancer growth, progression and metastasis. Br J Cancer 106: 1833-1841, 2012.

82. Barderas R, Bartolome RA, Fernandez-Acenero MJ, Torres S and Casal JI: High expression of IL-13 receptor $\alpha 2$ in colorectal cancer is associated with invasion, liver metastasis, and poor prognosis. Cancer Res 72: 2780-2790, 2012.

83. Formentini A, Braun P, Fricke H, Link KH, Henne-Bruns D and Kornmann M: Expression of interleukin-4 and interleukin-13 and their receptors in colorectal cancer. Int J Colorectal Dis 27 1369-1376, 2012

84.Liang J, Li Y, Liu X, Xu X and Zhao Y: Relationship between cytokine levels and clinical classification of gastric cancer. Asian Pac J Cancer Prev 12: 1803-1806, 2011.

85. Leushacke M, Sporle R, Bernemann C, et al: An RNA interference phenotypic screen identifies a role for FGF signals in colon cancer progression. PLoS One 6: e23381, 2011.

86.Zhao WM, Wang L, Park H, et al: Monoclonal antibodies to fibroblast growth factor receptor 2 effectively inhibit growth of gastric tumor xenografts. Clin Cancer Res 16: 5750-5758, 2010

87. Hwang TL, Lee LY, Wang CC, Liang Y, Huang SF and Wu CM CCL7 and CCL21 overexpression in gastric cancer is associated with lymph node metastasis and poor prognosis. World J Gastroenterol 18: 1249-1256, 2012.

88. Cambien B, Richard-Fiardo P, Karimdjee BF, et al: CCL5 neutralization restricts cancer growth and potentiates the targeting of PDGFR $\beta$ in colorectal carcinoma. PLoS One 6 e28842, 2011.

89. Chen HJ, Edwards R, Tucci S, et al: Chemokine 25-induced signaling suppresses colon cancer invasion and metastasis. J Clin Invest 122: 3184-3196, 2012.

90. Heckmann D, Laufs S, Maier P, et al: A lentiviral CXCR4 overexpression and knockdown model in colorectal cancer cell lines reveals plerixafor-dependent suppression of SDF-1 $\alpha$-induced migration and invasion. Onkologie 34: 502-508, 2011.

91. Margolin DA, Silinsky J, Grimes C, et al: Lymph node stromal cells enhance drug-resistant colon cancer cell tumor formation through SDF-1 $\alpha /$ CXCR4 paracrine signaling. Neoplasia 13 874-886, 2011

92. Skeen VR, Paterson I, Paraskeva C and Williams AC: TGF- $\beta 1$ signalling, connecting aberrant inflammation and colorectal tumorigenesis. Curr Pharm Des 18: 3874-3888, 2012.

93. Ananiev J, Gulubova M, Tchernev G, et al: Relation between transforming growth factor- $\beta 1$ expression, its receptor and clinicopathological factors and survival in HER2-negative gastric cancers. Wien Klin Wochenschr 123: 668-673, 2011.

94. Terashima M, Kitada K, Ochiai A, et al: Impact of expression of human epidermal growth factor receptors EGFR and ERBB2 on survival in stage II/III gastric cancer. Clin Cancer Res 18 5992-6000, 2012

95.Liu Y, Li Q and Zhu L: Expression of the hepatocyte growth factor and c-Met in colon cancer: correlation with clinicopathological features and overall survival. Tumori 98: 105-112, 2012.

96. Yang X, Takano Y and Zheng HC: The pathobiological features of gastrointestinal cancers (Review). Oncol Lett 3: 961-969, 2012.

97. Kim JY, Bae BN, Kwon JE, Kim HJ and Park K: Prognostic significance of epidermal growth factor receptor and vascular endothelial growth factor receptor in colorectal adenocarcinoma. APMIS 119: 449-459, 2011.
98. Tsai HL, Yang IP, Lin CH, et al: Predictive value of vascular endothelial growth factor overexpression in early relapse of colorectal cancer patients after curative resection. Int J Colorectal Dis 28: 415-424, 2013.

99. Hasan MR, Ho SH, Owen DA and Tai IT: Inhibition of VEGF induces cellular senescence in colorectal cancer cells. Int J Cancer 129: 2115-2123, 2011

100. Morales-Gutierrez C, Abad-Barahona A, Moreno-Gonzalez E, Enriquez de Salamanca R and Vegh I: Tumour VEGF/non tumour VEGF protein expression ratio as a biomarker for survival in colorectal cancer patients. Eur J Surg Oncol 37: 526-531, 2011

101. Sun P, Yu H, Zhang WQ, Hu M and Lv R: Lentivirus-mediated siRNA targeting VEGF inhibits gastric cancer growth in vivo. Oncol Rep 28: 1687-1692, 2012.

102. Li M, Liu F, Sun P, et al: Correlations between serum levels of vascular endothelial growth factor and endostatin with clinical pathological characteristics of patients with gastrointestinal cancers. Hepatogastroenterology 59: 1865-1868, 2012.

103. Villarejo-Campos P, Padilla-Valverde D, Martin RM, et al: Serum VEGF and VEGF-C values before surgery and after postoperative treatment in gastric cancer. Clin Transl Oncol 15: 265-270, 2013

104.Zhou Y, Li N, Zhuang W and Wu X: Vascular endothelial growth factor (VEGF) gene polymorphisms and gastric cancer risk in a Chinese Han population. Mol Carcinog 50: 184-188, 2011.

105. Zhang M, Zhu GY, Gao HY, Zhao SP and Xue Y: Expression of tissue levels of matrix metalloproteinases and tissue inhibitors of metalloproteinases in gastric adenocarcinoma. J Surg Oncol 103: 243-247, 2011.

106. Kosaka Y, Mimori K, Fukagawa T, et al: Clinical significance of molecular detection of matrix metalloproteinase- 1 in bone marrow and peripheral blood in patients with gastric cancer. Ann Surg Oncol 19 (Suppl 3): S430-S437, 2012.

107. Lu ZH, Fang YJ, Wu XJ, Pan ZZ and Wan DS: Expression of matrix metalloproteinase 1 in tissue of colon carcinoma and its clinical prognostic significance. Zhonghua Yi Xue Za Zhi 91: 2895-2898, 2011 (In Chinese).

108. Noh S, Jung JJ, Jung M, et al: MMP-2 as a putative biomarker for carcinomatosis in gastric cancer. Hepatogastroenterology 58: 2015-2019, 2011.

109. Kryczka J, Stasiak M, Dziki L, Mik M, Dziki A and Cierniewski C: Matrix metalloproteinase-2 cleavage of the $\beta 1$ integrin ectodomain facilitates colon cancer cell motility. J Biol Chem 287: 36556-36566, 2012.

110. Yang B, Su K, Gao J and Rao Z: Expression and prognostic value of matrix metalloproteinase-7 in colorectal cancer. Asian Pac J Cancer Prev 13: 1049-1052, 2012.

111. Bi Z, Dong LD and Gu XM: Clinical significance of MMP-7 and PTEN expression in colorectal cancer. Hepatogastroenterology 60: 32-36, 2012.

112. Fanelli MF, Chinen LT, Begnami MD, et al: The influence of transforming growth factor- $\alpha$, cyclooxygenase-2, matrix metalloproteinase (MMP)-7, MMP-9 and CXCR4 proteins involved in epithelial-mesenchymal transition on overall survival of patients with gastric cancer. Histopathology 61: 153-161, 2012.

113. Sena P, Mariani F, Marzona L, et al: Matrix metalloproteinases 15 and 19 are stromal regulators of colorectal cancer development from the early stages. Int J Oncol 41: 260-266, 2012.

114. Inagaki D, Oshima T, Yoshihara K, et al: Overexpression of tissue inhibitor of metalloproteinase-1 gene correlates with poor outcomes in colorectal cancer. Anticancer Res 30: 4127-4130, 2010.

115. Yoshikawa T, Cho H, Tsuburaya A and Kobayashi O: Impact of plasma tissue inhibitor of metalloproteinase-1 on long-term survival in patients with gastric cancer. Gastric Cancer 12: 31-36, 2009

116. Watanabe M, Takahashi Y, Ohta T, Mai M, Sasaki T and Seiki M: Inhibition of metastasis in human gastric cancer cells transfected with tissue inhibitor of metalloproteinase 1 gene in nude mice. Cancer 77 (Suppl 8): 1676-1680, 1996.

117. Miyagi M, Aoyagi K, Kato S and Shirouzu K: The TIMP-1 gene transferred through adenovirus mediation shows a suppressive effect on peritoneal metastases from gastric cancer. Int J Clin Oncol 12: 17-24, 2007

118. Nuovo GJ, Macconnell PB, Simsir A, Valea F and French DL: Correlation of the in situ detection of polymerase chain reaction-amplified metalloproteinase complementary DNAs and their inhibitors with prognosis in cervical carcinoma. Cancer Res 55: 267-275, 1995. 
119. Li BS, Zhao YL, Guo G, et al: Plasma microRNAs, miR-223, miR-21 and miR-218, as novel potential biomarkers for gastric cancer detection. PLoS One 7: e41629, 2012.

120.Li C, Li JF, Cai Q, et al: MiRNA-199a-3p in plasma as a potential diagnostic biomarker for gastric cancer. Ann Surg Oncol: Sep 7 , 2012 (Epub ahead of print).

121.Zhang X, Cui L, Ye G, et al: Gastric juice microRNA-421 is a new biomarker for screening gastric cancer. Tumour Biol 33: 2349-2355, 2012.

122. Wang M, Gu H, Wang S, et al: Circulating miR-17-5p and miR-20a: Molecular markers for gastric cancer. Mol Med Rep 5: $1514-1520,2012$.

123. Valladares-Ayerbes M, Reboredo M, Medina-Villaamil V, et al: Circulating miR-200c as a diagnostic and prognostic biomarker for gastric cancer. J Transl Med 10: 186, 2012.

124. Kanaan Z, Rai SN, Eichenberger MR, et al: Plasma miR-21: a potential diagnostic marker of colorectal cancer. Ann Surg 256: 544-551, 2012.

125.Nugent M, Miller N and Kerin MJ: Circulating miR-34a levels are reduced in colorectal cancer. J Surg Oncol 106: 947-952, 2012.

126. Inoue T, Inuma H, Ogawa E, Inaba T and Fukushima R: Clinicopathological and prognostic significance of microRNA-107 and its relationship to DICER1 mRNA expression in gastric cancer. Oncol Rep 27: 1759-1764, 2012.

127.Li J, Guo Y, Liang X, et al: MicroRNA-223 functions as an oncogene in human gastric cancer by targeting FBXW7/hCdc4. J Cancer Res Clin Oncol 138: 763-774, 2012.
128. Xu XM, Qian JC, Deng ZL, et al: Expression of miR-21, miR-31, miR-96 and miR-135b is correlated with the clinical parameters of colorectal cancer. Oncol Lett 4: 339-345, 2012.

129. Zhou T, Zhang G, Liu Z, Xia S and Tian H: Overexpression of miR-92a correlates with tumor metastasis and poor prognosis in patients with colorectal cancer. Int J Colorectal Dis 28: 19-24, 2013.

130.Hur K, Toiyama Y, Takahashi M, et al: MicroRNA-200c modulates epithelial-to-mesenchymal transition (EMT) in human colorectal cancer metastasis. Gut: Jul 10, 2012 (Epub ahead of print).

131. Li CL, Nie H, Wang M, et al: microRNA-155 is downregulated in gastric cancer cells and involved in cell metastasis. Oncol Rep 27: 1960-1966, 2012

132. Li Z, Cao Y, Jie Z, et al: miR-495 and miR-551a inhibit the migration and invasion of human gastric cancer cells by directly interacting with PRL-3. Cancer Lett 323: 41-47, 2012.

133. Ruzzo A, Graziano F, Vincenzi B, et al: High let-7a microRNA levels in KRAS-mutated colorectal carcinomas may rescue anti-EGFR therapy effects in patients with chemotherapyrefractory metastatic disease. Oncologist 17: 823-829, 2012.

134. Xu K, Liang X, Shen K, et al: MiR-222 modulates multidrug resistance in human colorectal carcinoma by down-regulating ADAM-17. Exp Cell Res 318: 2168-2177, 2012

135. Osawa S, Shimada Y, Sekine S, et al: MicroRNA profiling of gastric cancer patients from formalin-fixed paraffin-embedded samples. Oncol Lett 2: 613-619, 2011. 